\title{
To ascertain any differences in fetomaternal outcomes in induced and spontaneous labour among primiparous women delivering at term without an identified indication for induction
}

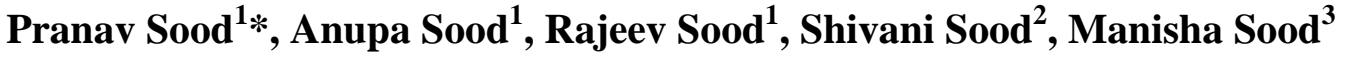

\author{
${ }^{1}$ Department of Obstetrics and Gynaecology, Indira Gandhi Medical College, Shimla, HP, India \\ ${ }^{2}$ Department of Pathology, Indira Gandhi Medical College, Shimla, HP, India \\ ${ }^{3}$ Department of Physiology, LTMMC, Sion, Mumbai, Maharashtra, India
}

Received: 30 October 2015

Accepted: 12 December 2015

*Correspondence:

Dr. Pranav Sood,

E-mail: pranavsood2031@rediffmail.com

Copyright: (c) the author(s), publisher and licensee Medip Academy. This is an open-access article distributed under the terms of the Creative Commons Attribution Non-Commercial License, which permits unrestricted non-commercial use, distribution, and reproduction in any medium, provided the original work is properly cited .

\begin{abstract}
Background: The objective of the stusy was to ascertain any differences in fetomaternal outcomes in induced and spontaneous labour among primiparous women delivering at term without an identified indication for induction.

Methods: This was a prospective study of 500 women with spontaneous labour and 204 women with induced labour who were delivered at 37 weeks to $40+6$ weeks gestation, all without identified medical and obstetrical indications for induction.

Results: Initial Bishop score in the induced group was low $(\leq 5)$ in $78 \%$ compared to $46 \%$ in spontaneous group $(\mathrm{p}<0.001)$. Mean duration of total labour $(9.1+4.42$ Vs $8+4.41$ hours $)$, first stage of labour $(8.5+2.3$ Vs $7.4+1.6$ hours $)$ was significantly short in induced group as compared to spontaneous group $(p<0.001)$. Induced subjects had higher incidence of caesarean delivery compared with spontaneous group $(\mathrm{p}=0.016)$, interestingly incidence of instrumental delivery was insignificantly higher in spontaneous group compared to induced group ( $7.8 \%$ vs. $3.9 \%$, $p=0.06)$, no difference was found regards second and third stage, duration of rupture of membranes, vaginal lacerations, 1 minute and 5 minute Apgar scores, admission to NICU and hospital stay.

Conclusions: Primipara who has spontaneous onset of labour the initial mean Bishop score is more compared to the subjects who have induced labour. The study demonstrated a significant increase in rate of caesarean section when Bishop Score was $\leq 5(\mathrm{p}=0.047)$. Compared to those with spontaneous labour, primiparas with induced labour are more likely to have short duration of labour specially the first stage and higher incidence of caesarean delivery.
\end{abstract}

Keywords: Induction of labour, Spontaneous labour, Caesarean delivery, Instrumental delivery, Neonatal outcome

\section{INTRODUCTION}

Induction of labour is the artificial initiation of labour before its spontaneous onset for the purpose of delivery of the fetoplacental unit. It is performed when the benefits of delivery outweigh the risks of continuing the pregnancy. ${ }^{1}$ Induction of labour is increasing in the U.S. The overall induction rate has increased from $9.5 \%$ in $1990-22.1 \%$ in $2004 .^{2}$ This increase in induction was mirrored by an increase in the caesarean section rate from $23 \%$ in $1990-30 \%$ in $2005 .^{3}$ Induction of labour that is not indicated for a medical reason, also termed elective induction of labour, appears to be rising as well and at a rate even more rapidly than that of the overall induction of labour. ${ }^{4}$ Many think that this intervention exposes the parturient patient and her baby to a cascade of related events each contributing its own hazards, the culmination of which is less favourable outcome than would be obtained if nature were allowed to follow its course. ${ }^{5,6}$ The commonly held dogma regarding induction of labour is that it increases the risk of cesarean delivery, which in turn is associated with a host of maternal and neonatal 
complications in present and future pregnancies. ${ }^{2}$ Although the literature on elective induction is limited, advantages and disadvantages have been described. ${ }^{6,7}$ Opinions differ regarding whether benefits outweigh the risks to mother and foetus during induction of labour. With this background knowledge, the present study was planned with an aim to compare the fetomaternal outcomes of spontaneous and induced labour in primiparas. It is hoped that the results of this study would add to the body of evidence on this subject.

\section{METHODS}

The study was conducted in the Department of Obstetrics and Gynecology, Kamla Nehru Hospital for mother and child, Indira Gandhi Medical College, Shimla, India for one year duration from $1^{\text {st }}$ June 2011 to $31^{\text {st }}$ May 2012 which included all primiparous women undergoing elective induction who fulfilled the following criteria: women willing to participate in study, primiparous women, gestational age between 37 completed weeks to $40+6$ weeks as determined by the last menstrual period/ positive pregnancy test at 5 weeks/ Per vaginal examination and/or by ultrasound scan in first trimester, singleton viable fetus, cephalic presentation, no contraindication to vaginal delivery.

\section{Exclusion criteria}

It included multiparous women, women with an intrauterine foetal death, known lethal anomaly, multifetal gestation, abnormal placentation, abnormal presentation, women with previous caesarean section or previous uterine surgery, women with all medical disorders, women with other obstetric complications like PIH and IUGR, any other indication for Caesarean delivery.

A complete history was taken as per pre-designed proforma. General physical and obstetrical examination was carried out. Per vaginal examination was done to know the Bishop score and adequacy of pelvis for vaginal delivery. The following observations were made in each parturient: duration of first stage, duration of second stage, duration of rupture of membranes, lacerations, mode of delivery (normal, instrumental, vaginal or caesarean), indication of caesarean delivery, third stage duration and any occurrence of PPH was noted.

Neonatal outcome was monitored in terms of any gross congenital anomaly, birth weight, sex, Apgar score at 1 and 5 minutes was noted and duration of hospital stay in nursery and neonatal deaths if any were noted.

Labour induction was commenced in accordance of hospital protocol as follows:

1. In unfavorable cervix (Bishop's score $\leq 5$ ), $25 \mu \mathrm{g}$ of misoprostol was placed in posterior fornix of vagina and repeated every 4 hourly to a maximum of five doses or till the women enter into active phase of labour or dinoprostone gel $0.5 \mathrm{mg}$ was instilled intracervically to a maximum of 2 doses 8 hours apart.

2. In favorable cervix (Bishop's score $\geq 6$ ), artificial rupture of membranes (ARM) was done and after 2 hours, oxytocin infusion was started if labour pains were inadequate and dose escalation was done if required according to geometrical progression.

Labour was monitored using WHO partogram in all the women.

Success of induction was defined as Normal vaginal delivery after induction of labour.

On the basis of onset of labour women were classified into two groups, first with spontaneous onset of labour and second with induced labour.

\section{Statistical analysis}

The percentage of each qualitative variable and the mean, standard deviation, minimum and maximum values for the quantitative variables were measured.

Data were entered into statistical software package SPSS version 17 and epi info. The t-test was used for quantitative data and Pearson Chi square or fisher's exact test was used for categorical databases. Multivariant logistic regression was performed using all the significant variables with $\mathrm{p}$ value $<0.05$ in the univariate test.

\section{RESULTS}

There were 6111 deliveries in Kamla Nehru State Hospital for Mother and Child, Indira Gandhi Medical College from $1^{\text {st }}$ June $2011-31^{\text {st }}$ May 2012 of which only 704 were included in the study which fulfilled the inclusion criteria. $500(71 \%)$ women had spontaneous labour (Group 1) and 204 (29\%) women had induced labour (Group 2). Induction was done with misoprostol, dinoprostone or oxytocin infusion.

Labour induction was commenced in accordance of hospital protocol (dinoprostone gel/misoprostol/ ARM + Oxytocin) and was monitored by WHO partogram. The outcome of labour, delivery and neonate's details were obtained and recorded from the clinical notes after delivery. When required operative interference was done to expedite instrumental vaginal delivery or caesarean delivery for the safety of the mother, baby or both. Data was analysed using t-test, Pearson-Chi square, fisher's exact test and multivariant logistic regression using epi info software and SPSS software.

Mean age of subjects were $25.2 \pm 3.4$ years in group 1 and $25.2 \pm 3.36$ years in group 2 ( $\mathrm{p}$ value 0.94$)$. Age wise both the groups matched each other (Table 1). 
Table 1: Age-wise distribution of the cases.

\begin{tabular}{|lllll|}
\hline $\begin{array}{l}\text { Age } \\
\text { (years) }\end{array}$ & \multicolumn{2}{l|}{ Group1 $(\mathbf{n = 5 0 0 )}$} & \multicolumn{2}{l|}{ Group $2(\mathbf{n = 2 0 4})$} \\
\hline$\leq 18$ & No & Percent & No & Percent \\
\hline $19-25$ & 284 & 56.8 & 1 & 0.5 \\
\hline $26-30$ & 186 & 37.2 & 118 & 57.8 \\
\hline $31-35$ & 23 & 4.6 & 73 & 35.8 \\
\hline$>35$ & 5 & 1 & 11 & 5.4 \\
\hline & Mean=25.2 & 1 & 0.5 \\
\hline & Min=18 & Mean=25.2 \\
& Max=40 & Min=18 \\
& S.D=3.4 & S.D $=3.36$ \\
\hline & P value: 0.94 & \\
\hline
\end{tabular}

Mean height was $155.47 \pm 3.8 \mathrm{cms}$ in group 1 and $155.8 \pm 2.95 \mathrm{cms}$ in group $2(\mathrm{p}=0.27)$, and this was comparable (Table 2).

Table 2: Height-wise distribution of the subjects.

\begin{tabular}{|lllll|}
\hline $\begin{array}{l}\text { Height } \\
(\mathrm{cm})\end{array}$ & \multicolumn{2}{l|}{ Group $1(\mathrm{n}=500)$} & Group $2(\mathrm{n}=\mathbf{2 0 4})$ \\
\hline$\leq 150$ & No & Percent & No & Percent \\
\hline $150.1-155$ & 242 & 7.4 & 10 & 4.9 \\
\hline$>155.1-160$ & 167 & 33.4 & 100 & 49 \\
\hline$\geq 160$ & 54 & 10.8 & 10 & 4.9 \\
\hline & Mean=155.47 & Mean=155.8 \\
& Min=146 & Min=146 \\
& Max=169 & Max=169 \\
& S.D=3.8 & S.D =2.95 \\
\hline & P value $=0.27$ & \\
\hline
\end{tabular}

Mean weight was $59.26 \pm 4.9 \mathrm{kgs}$ in group 1 and $59.17 \pm 3.38 \mathrm{kgs}$ in group 2 (p value 0.8 ) and was comparable (Table 3).

Table 3: Weight wise distribution.

\begin{tabular}{|c|c|c|c|c|}
\hline \multirow[t]{2}{*}{$\begin{array}{l}\text { Weight } \\
\text { (kg) }\end{array}$} & \multicolumn{2}{|c|}{$\begin{array}{l}\text { Group } 1 \\
(n=500)\end{array}$} & \multicolumn{2}{|c|}{$\begin{array}{l}\text { Group } \\
2(n=204)\end{array}$} \\
\hline & No & Percent & No & Percent \\
\hline $45-50$ & 5 & 1 & 2 & 1 \\
\hline $50.1-55$ & 81 & 16.2 & 12 & 5.9 \\
\hline $55.1-60$ & 281 & 56.2 & 139 & 68.1 \\
\hline $60.1-65$ & 57 & 11.4 & 38 & 18.6 \\
\hline $65.1-70$ & 67 & 13.4 & 12 & 5.9 \\
\hline$>70$ & 9 & 1.8 & 1 & 0.5 \\
\hline & \multicolumn{2}{|c|}{$\begin{array}{l}\text { Mean }=59.26 \\
\text { Min= } 45 \\
\text { Max= } 75 \\
\text { S.D }=4.9\end{array}$} & \multicolumn{2}{|c|}{$\begin{array}{l}\text { Mean=59.17 } \\
\text { Min=45 } \\
\text { Max=77 } \\
\text { S.D=3.38 }\end{array}$} \\
\hline \multicolumn{3}{|c|}{$P$ value $=0.804$} & & \\
\hline
\end{tabular}

Mean BMI in group 1 was $24.5 \pm 1.6 \mathrm{~kg} / \mathrm{m}^{2}$ and in group 2 was $24.37 \pm 1.34 \mathrm{~kg} / \mathrm{m}^{2} \quad(\mathrm{p}=0.336)$, both the groups matched each other (Table 4).
Table 4: Comparison of BMI.

\begin{tabular}{|c|c|c|c|c|}
\hline \multirow[t]{2}{*}{ BMI } & \multicolumn{2}{|c|}{ Group1 (n=500) } & \multicolumn{2}{|c|}{$\begin{array}{l}\text { Group } 2 \\
(n=204)\end{array}$} \\
\hline & No & Percent & No & Percent \\
\hline$<19$ & 2 & 0.4 & 0 & 0 \\
\hline $19-25$ & 316 & 63.2 & 145 & 71.1 \\
\hline $25.1-30$ & 180 & 36 & 59 & 28.9 \\
\hline $30.1-35$ & 2 & 0.4 & 0 & 0 \\
\hline & \multicolumn{2}{|c|}{ Mean $=24.5$} & \multicolumn{2}{|c|}{ Mean $=24.37$} \\
\hline & \multicolumn{2}{|c|}{$\operatorname{Min}=18.5$} & \multicolumn{2}{|c|}{$\operatorname{Min}=18.5$} \\
\hline & \multicolumn{2}{|c|}{$\operatorname{Max}=30.3$} & \multicolumn{2}{|c|}{$\operatorname{Max}=30.3$} \\
\hline & \multicolumn{2}{|c|}{ S.D $=1.6$} & \multicolumn{2}{|c|}{ S.D $=1.34$} \\
\hline & \multicolumn{4}{|c|}{$P$ value $=0.336$} \\
\hline
\end{tabular}

The mean gestational age was 39.23 weeks and 39.3 weeks in group 1 and group 2 respectively $(\mathrm{p}=0.54)$ which was found to be almost same in both groups (Table 5).

Table 5: Distribution according to gestational age.

\begin{tabular}{|lllc|}
\hline Gestational age & \multicolumn{2}{c|}{ Primigravida $(\mathrm{n}=704)$} & P \\
& No & Percent & value \\
\hline $37^{0 / 7}-38^{0 / 6}$ weeks & 205 & 29.1 & 0.54 \\
\hline $39^{0 / 7}-40^{0 / 6}$ weeks & 499 & 70.9 & \\
\hline
\end{tabular}

Subjects with Bishop score $\leq 5$ had higher incidence of caesarean delivery compared with those who had Bishop $\geq 6(p=0.047)$ (Table 6). It was concluded that the initial Bishop score in the induced group was low $(\leq 5)$ in a very high number of subjects $(78 \%$ mean Bishop score $3.8 \pm 1.2$ ) compared to $46 \%$ in spontaneous group (mean Bishop score $6 \pm 1.5)(\mathrm{p}<0.001)$ (Table 7). The caesarean rate in induced group was significantly high $45.1 \% \mathrm{Vs}$ $35.4 \%(\mathrm{p}=0.02)($ Table 13$)$.

Table 6: Relationship of Bishop score with mode of delivery.

\begin{tabular}{|llcccc|}
\hline $\begin{array}{l}\text { Bishop } \\
\text { score }\end{array}$ & \multicolumn{2}{l}{$\begin{array}{l}\text { Vaginal delivery } \\
(\mathbf{n = 4 3 5 )}\end{array}$} & \multicolumn{2}{l|}{$\begin{array}{l}\text { Caesarean } \\
\text { section }(\mathbf{n = 2 6 9 )}\end{array}$} & $\begin{array}{l}\text { P } \\
\text { value }\end{array}$ \\
\cline { 1 - 5 } & No & Percent & No & Percent & \\
\hline$\leq 5$ & 227 & 52.2 & 161 & 59.8 & \multirow{2}{*}{0.047} \\
\cline { 1 - 5 }$\geq 6$ & 208 & 47.8 & 108 & 40.2 & \\
\hline
\end{tabular}

Table 7: Comparison of Bishops score and onset of labour.

\begin{tabular}{|lllll|l|}
\hline $\begin{array}{l}\text { Bishop } \\
\text { score }\end{array}$ & \multicolumn{2}{l|}{$\begin{array}{l}\text { Vaginal delivery } \\
(\mathbf{n}=\mathbf{4 3 5})\end{array}$} & \multicolumn{2}{c|}{$\begin{array}{c}\text { Caesarean } \\
\text { section }(\mathbf{n = 2 6 9 )}\end{array}$} & p value \\
& No & Percent & No & Percent & \\
\hline$\leq 5$ & 230 & 46 & 159 & 78 & $<.001$ \\
\hline$\geq 6$ & 270 & 54 & 45 & 22 & $<.001$ \\
\hline
\end{tabular}

Mean duration of total labour $(9.1 \pm 4.42 \mathrm{Vs} 8 \pm 4.41)$ hours (Table 8$)$ and first stage of labour (8.5 \pm 2.3 Vs $7.4 \pm 1.6)$ 
hours (Table 9) was significantly short in induced group as compared to spontaneous group $(\mathrm{p}<0.001)$.

Table 8: Comparison of total duration of labour (hours) between the two groups.

\begin{tabular}{|cccc|}
\hline Number & $\begin{array}{l}\text { Group 1 } \\
(\text { mean+SD) } \\
\text { hours }\end{array}$ & $\begin{array}{l}\text { Group 2 } \\
(\text { mean+SD) } \\
\text { hours }\end{array}$ & p value \\
\hline 435 & $9.1 \pm 4.42$ & $8 \pm 4.41$ & $<0.001$ \\
\hline
\end{tabular}

Table 9: Comparison of duration of first stage of labour (hours).

\begin{tabular}{|cccc|} 
Number & $\begin{array}{c}\text { Group 1 } \\
(\text { mean }+ \text { SD }) \\
\text { hours }\end{array}$ & $\begin{array}{l}\text { Group 2 } \\
(\text { mean } \pm \text { SD })\end{array}$ & p value \\
hours & \\
\hline 435 & $8.5 \pm 2.3$ & $7.4 \pm 1.6$ & $<0.001$ \\
\hline
\end{tabular}

Duration of second stage of labour was comparable between the two groups (Table 10).

Table 10: Comparison of duration of second stage of labour (minutes).

\begin{tabular}{|ccc|c|}
\hline Number & $\begin{array}{c}\text { Group 1 } \\
(\text { mean+SD) min }\end{array}$ & $\begin{array}{c}\text { Group 2 } \\
(\text { mean+SD) min }\end{array}$ & $\begin{array}{c}\text { p } \\
\text { value }\end{array}$ \\
\hline 435 & $36 \pm 16.2$ & $36.48 \pm 13$ & 0.782 \\
\hline
\end{tabular}

No significant difference was found between the two groups with respect to the duration of third stage (Table 11) and duration of rupture of membranes (Table 12).

Table 11: Comparison of duration of third stage of labour (minutes).

\begin{tabular}{|c|c|c|c|}
\hline Parameters & $\begin{array}{c}\text { Group } 1 \\
(\text { mean+SD) } \\
\text { mins }\end{array}$ & $\begin{array}{c}\text { Group } 2 \\
(\text { mean } \pm \text { SD) } \\
\text { mins }\end{array}$ & $\underset{\text { value }}{\mathbf{p}}$ \\
\hline $\begin{array}{l}\text { Third stage } \\
\text { duration (mins) }\end{array}$ & $3.28 \pm 0.58$ & $3.26 \pm 0.48$ & 0.789 \\
\hline
\end{tabular}

Table 12: Comparison of duration of rupture of membranes (hours).

\begin{tabular}{|cccc|}
\hline Number & $\begin{array}{c}\text { Group 1 } \\
\left(\begin{array}{c}\text { mean+SD } \\
\text { hours }\end{array}\right.\end{array}$ & $\begin{array}{c}\text { Group 2 } \\
(\text { mean+SD }) \\
\text { hours }\end{array}$ & $\begin{array}{l}\text { p } \\
\text { value }\end{array}$ \\
\hline $\mathbf{7 0 4}$ & $4.54 \pm 1.6$ & $4.45+ \pm 1.3$ & 0.605 \\
\hline
\end{tabular}

Caesarean section rate was found to be higher in induced group $(45.1 \%)$ compared to $(35.4 \%)$ spontaneous group $(\mathrm{p}=0.02)$. Instrumental delivery rate was higher in spontaneous group (7.8\%) as compared to induced group $(3.9 \%)$ but statistically insignificant $(\mathrm{p}=0.06)($ Table 13$)$.

Most common indication for caesarean was found to be fetal distress. Interestingly this was significantly higher in spontaneous group as compared to induced group $(\mathrm{p}<0.001)$. Dystocia responsible for caesarean section was found to be higher in induced group but it was statistically insignificant $(\mathrm{p}=0.1)($ Table 14).

Table 13: Comparison of mode of delivery between two groups.

\begin{tabular}{|c|c|c|c|c|c|}
\hline \multirow[t]{2}{*}{$\begin{array}{l}\text { Mode of } \\
\text { delivery }\end{array}$} & \multicolumn{2}{|c|}{$\begin{array}{l}\text { Group } 1 \\
(n=500)\end{array}$} & \multicolumn{2}{|c|}{$\begin{array}{l}\text { Group } 2 \\
(n=204)\end{array}$} & \multirow[t]{2}{*}{$\begin{array}{l}p \\
\text { value }\end{array}$} \\
\hline & No & Percent & No & Percent & \\
\hline $\begin{array}{l}\text { Normal } \\
\text { vaginal } \\
\text { delivery }\end{array}$ & 284 & 56.8 & 104 & 51 & 0.16 \\
\hline $\begin{array}{l}\text { Instrumental } \\
\text { delivery }\end{array}$ & 39 & 7.8 & 8 & 3.9 & 0.06 \\
\hline $\begin{array}{l}\text { Caesarean } \\
\text { section }\end{array}$ & 177 & 35.4 & 92 & 45.1 & 0.02 \\
\hline
\end{tabular}

Table 14: Comparison of Indication of caesarean by onset of labour.

\begin{tabular}{|llllll|}
\hline $\begin{array}{l}\text { Indication } \\
\text { of } \\
\text { caesarean }\end{array}$ & \multicolumn{2}{l}{$\begin{array}{l}\text { Group 1 } \\
(\mathbf{n}=500)\end{array}$} & \multicolumn{2}{l|}{$\begin{array}{l}\text { Group 2 } \\
(\mathrm{n}=204)\end{array}$} & $\begin{array}{l}\text { p } \\
\text { value }\end{array}$ \\
\cline { 2 - 5 } & No & Percent & No & Percent & \\
\hline $\begin{array}{l}\text { Fetal } \\
\text { distress }\end{array}$ & 161 & 32.2 & 60 & 29.4 & $<0.001$ \\
\hline Dystocia & 14 & 2.8 & 13 & 6.4 & 0.1 \\
\hline $\begin{array}{l}\text { Failed } \\
\text { induction }\end{array}$ & 0 & 0 & 18 & 8.8 & NA \\
\hline Others & 2 & 0.4 & 1 & 0.5 & 0.97 \\
\hline
\end{tabular}

There was no significant difference between the two groups as far as postpartum haemorrhage was concerned $(\mathrm{p}=0.3)$ (Table 15). Both groups were comparable as regards lacerations $(p>0.05)$ (Table 16), placental weight $(\mathrm{p}=0.162), 1$ and 5 minute apgar score $(\mathrm{p}=0.51)$ (Table 17 and 18).

Table 15: Comparison of incidence of PPH between the two groups.

\begin{tabular}{|c|c|c|c|c|c|}
\hline & \multicolumn{2}{|c|}{$\begin{array}{l}\text { Group } 1 \\
(n=500)\end{array}$} & \multicolumn{2}{|c|}{$\begin{array}{l}\text { Group } 2 \\
(n=204)\end{array}$} & \multirow[t]{2}{*}{ p value } \\
\hline & No & Percent & No & Percent & \\
\hline PPH & 6 & 1.2 & 4 & 1.9 & 0.3 \\
\hline
\end{tabular}

Table 16: Comparison of lacerations between the subjects.

\begin{tabular}{|lccccc|}
\hline Lacerations & \multicolumn{2}{c}{$\begin{array}{c}\text { Group 1 } \\
(\mathrm{n}=500)\end{array}$} & \multicolumn{2}{c|}{$\begin{array}{c}\text { Group 2 } \\
(\mathrm{n}=204)\end{array}$} & $\begin{array}{l}\text { v } \\
\text { value }\end{array}$ \\
\cline { 1 - 5 } No & Percent & No & Percent & \\
\hline Perineal tear & 9 & 1.8 & 3 & 1.5 & 0.7 \\
\hline Cervical & 7 & 1.4 & 1 & 1.5 & 0.46 \\
\hline Vaginal & 6 & 1.2 & 2 & 1 & 0.77 \\
\hline
\end{tabular}


Table 17: Comparison of 1 minute Apgar score between the two groups.

\begin{tabular}{cccccc|}
\hline $\begin{array}{c}1 \text { minute } \\
\begin{array}{c}\text { Apgar } \\
\text { score }\end{array}\end{array}$ & \multicolumn{2}{c}{$\begin{array}{c}\text { Group 1 } \\
(\mathbf{n}=500)\end{array}$} & \multicolumn{2}{c|}{$\begin{array}{c}\text { Group 2 } \\
(\mathbf{n}=204)\end{array}$} & $\begin{array}{l}\text { p } \\
\text { value }\end{array}$ \\
\hline$<7$ & 5 & Percent & No & Percent & \\
\hline$<$ & 1 & 2 & 1 & 0.51 \\
\hline
\end{tabular}

Table 18 : Comparison of 5 minute Apgar score between the two groups.

\begin{tabular}{cccccc|}
$\begin{array}{c}5 \text { minute } \\
\begin{array}{c}\text { Apgar } \\
\text { score }\end{array}\end{array}$ & \multicolumn{2}{c}{$\begin{array}{c}\text { Group 1 } \\
(\mathrm{n}=500)\end{array}$} & \multicolumn{2}{c|}{$\begin{array}{c}\text { Group 2 } \\
(\mathrm{n}=204)\end{array}$} & $\begin{array}{l}\text { p } \\
\text { value }\end{array}$ \\
\hline$<7$ & 5 & Percent & No & Percent & \\
\hline & 1 & 2 & 1 & 0.51 \\
\hline
\end{tabular}

Table 19: Comparison of neonatal birth weight in two groups.

\begin{tabular}{|c|c|c|c|c|c|}
\hline \multirow[t]{2}{*}{$\begin{array}{l}\text { Birth } \\
\text { weight }(\mathrm{g})\end{array}$} & \multicolumn{2}{|c|}{$\begin{array}{l}\text { Group } 1 \\
(\mathrm{n}=500)\end{array}$} & \multicolumn{2}{|c|}{$\begin{array}{l}\text { Group } 2 \\
(\mathrm{n}=204)\end{array}$} & \multirow[t]{2}{*}{$\begin{array}{l}p \\
\text { value }\end{array}$} \\
\hline & No & Percent & No & Percent & \\
\hline$<2000$ & 6 & 1.2 & 6 & 2.9 & 0.10 \\
\hline $2000-2499$ & 132 & 26.4 & 39 & 19.1 & $<.001$ \\
\hline $2500-2999$ & 254 & 50.8 & 95 & 46.6 & 0.3 \\
\hline $3000-3499$ & 92 & 18.4 & 53 & 26.0 & 0.02 \\
\hline \multirow[t]{3}{*}{$3500-3999$} & 16 & 3.2 & 11 & 5.4 & 0.17 \\
\hline & \multicolumn{2}{|c|}{$\begin{array}{l}\text { Mean=2797 } \\
\text { Min }=1800 \\
M a x=4300 \\
\text { SD }=37\end{array}$} & \multicolumn{2}{|c|}{$\begin{array}{l}\text { Mean }=2876 \\
M i n=1800 \\
M a x=4000 \\
\text { SD }=41\end{array}$} & \\
\hline & \multicolumn{2}{|c|}{$\mathrm{p}$ value $=0.01$} & & & \\
\hline
\end{tabular}

Table 20: Comparison of admission to neonatal ward between the two groups.

\begin{tabular}{cccccc}
$\begin{array}{c}\text { Admission } \\
\begin{array}{c}\text { to } \\
\text { neonatal }\end{array}\end{array}$ & $\begin{array}{c}\text { Group 1 } \\
(\mathrm{n}=500)\end{array}$ & \multicolumn{2}{c}{$\begin{array}{c}\text { Group 2 } \\
(\mathrm{n}=204)\end{array}$} & $\begin{array}{c}\mathrm{p} \\
\text { value }\end{array}$ \\
ward & No & Percent & No & Percent & \\
\hline
\end{tabular}

Table 21: Comparison of indications of admission to neonatal ward.

\begin{tabular}{|llllll|}
\hline $\begin{array}{l}\text { Admission } \\
\text { to neonatal }\end{array}$ & \multicolumn{2}{l}{$\begin{array}{l}\text { Group } 1 \\
(\mathbf{n}=500)\end{array}$} & \multicolumn{2}{l}{$\begin{array}{l}\text { Group 2 } \\
(\mathbf{n}=204)\end{array}$} & $\begin{array}{l}\text { p } \\
\text { value }\end{array}$ \\
\cline { 2 - 5 } & No & Percent & No & Percent & \\
\hline Meconeum & 18 & 3.6 & 8 & 3.9 & 0.98 \\
\hline $\begin{array}{l}\text { FHR } \\
\text { abnormality }\end{array}$ & 10 & 2 & 4 & 1.96 & 0.84 \\
\hline $\begin{array}{l}\text { Depressed } \\
\text { infant }\end{array}$ & 3 & 0.6 & 2 & 0.98 & 0.63 \\
\hline $\begin{array}{l}\text { Respiratory } \\
\text { difficulty }\end{array}$ & 3 & 0.6 & 1 & 0.5 & 0.8 \\
\hline
\end{tabular}

Mean birth weight in induced group was found to be significantly higher than in spontaneous group $2.876 \pm 0.41 \mathrm{Kg}$ Vs $2.797 \pm 0.37 \mathrm{Kg}(\mathrm{p}=0.01)$ (Table 19).

Both groups were comparable in terms of admission $(\mathrm{p}=0.8)$ (Table 20) and indications of admission to neonatal ward (Table 21), duration of hospital stay (Table 22). There were no neonatal deaths in any of the groups during the study period.

Table 22: Comparison of duration of hospital stays between the two groups.

\begin{tabular}{|llllll|}
\hline $\begin{array}{l}\text { Duration } \\
\text { of hospital }\end{array}$ & \multicolumn{2}{l}{$\begin{array}{l}\text { Group } 1 \\
(\mathbf{n}=500)\end{array}$} & \multicolumn{2}{l}{$\begin{array}{l}\text { Group 2 } \\
(\mathbf{n}=204)\end{array}$} & $\begin{array}{l}\text { p } \\
\text { value }\end{array}$ \\
\cline { 2 - 6 } stay & No & Percent & No & Percent & \\
\hline 0-48 hours & 480 & 96 & 196 & 96.1 & 0.96 \\
\hline 7 days & 13 & 2.6 & 5 & 2.45 & 0.9 \\
\hline >7 days & 7 & 1.4 & 3 & 1.47 & 0.94 \\
\hline
\end{tabular}

\section{DISCUSSION}

In the present study mean Bishop Score in group 1 was $6 \pm 1.5$ and $3.8 \pm 1.2$ in group 2. The present study is comparable to Johnson DP et al (2003) where mean

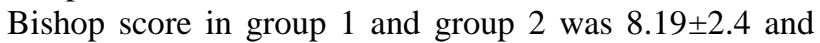
$5.3 \pm 2.3$ respectively and Vrouenraets FPJM et al (2005) (7.9 \pm 2.6 and $3.2 \pm 2.3$ in group 1 and 2 respectively). ${ }^{8,9}$ These studies demonstrated a significant difference in Bishop score between the two groups among primipara subjects. From the above studies including the present study it is concluded that in primiparas if the patient has spontaneous onset of labour the mean Bishop score is more compared to the subjects who have induced labour (Table 23).

Table 23: Bishop score distribution.

\begin{tabular}{|c|c|c|c|}
\hline Author & $\begin{array}{l}\text { Group } 1 \\
(\text { mean+SD) }\end{array}$ & $\begin{array}{l}\text { Group } 2 \\
(\text { mean+SD) }\end{array}$ & $\begin{array}{l}\text { p } \\
\text { Value }\end{array}$ \\
\hline $\begin{array}{l}\text { Johnson DP et al } \\
(2003)^{8}\end{array}$ & $8.19 \pm 2.4$ & $5.3 \pm 2.3$ & $<0.001$ \\
\hline $\begin{array}{l}\text { Vrouenraets } \text { FPJM } \\
\text { et al }(2005)^{9}\end{array}$ & $7.9 \pm 2.6$ & $3.2 \pm 2.3$ & $<0.001$ \\
\hline Present study & $6.3 \pm 1.8$ & $3.8 \pm 1.8$ & $<0.001$ \\
\hline
\end{tabular}

Johnson DP et al (2003) observed that the caesarean delivery rate was $31.5 \%$ in primipara subjects with Bishop score $<5$ and $18.1 \%$ among subjects with Bishop score $\geq 5$ at the time of induction ${ }^{8}$ and Vrouenraets FPJM et al (2005) reported caesarean delivery rate of $25 \%$ in primipara subjects with Bishop score $\leq 5,13.6 \%$ in those with Bishop score 6-8 and 6.2\% in subjects with Bishop score $>9$ at the time of induction. ${ }^{9}$ These studies demonstrated a highly significant increase in rate of caesarean section when Bishop score was $\leq 5(\mathrm{p}<0.001)$. In the present study caesarean delivery rate was $59.8 \%$ among subjects who were induced with Bishop score $\leq 5$ compared to $40.2 \%$ in those whose Bishop score was $\geq 6$ 
at the time of induction and the difference was found to be statistically significant ( $\mathrm{p}=0.047$ ) (Table 24).

Table 24: Relationship between Bishop score and caesarean delivery.

\begin{tabular}{|c|c|c|c|}
\hline Author & $\begin{array}{l}\text { Bishop } \\
\text { score }\end{array}$ & $\begin{array}{l}\text { Caesarean } \\
\text { rate }\end{array}$ & P value \\
\hline \multirow{2}{*}{$\begin{array}{l}\text { Johnson DP } \\
\text { et al }(2003)^{8}\end{array}$} & $<5$ & $31.5 \%$ & \multirow[t]{2}{*}{$<0.001$} \\
\hline & $\geq 5$ & $18.1 \%$ & \\
\hline \multirow{3}{*}{$\begin{array}{l}\text { Vrouenraets } \\
\text { FPJM et al } \\
(2005)^{9}\end{array}$} & $\leq 5$ & $25 \%$ & \multirow[t]{3}{*}{$<0.001$} \\
\hline & $6-8$ & $13.6 \%$ & \\
\hline & $>9$ & $6.2 \%$ & \\
\hline \multirow{2}{*}{$\begin{array}{l}\text { Present } \\
\text { study }\end{array}$} & $\leq 5$ & $41.4 \%$ & \multirow[t]{2}{*}{0.05} \\
\hline & $\geq 6$ & $34.3 \%$ & \\
\hline
\end{tabular}

The present study is comparable to Smith LP et al (1984) (10.9 hours in group 1 and 8.6 hours in group 2) $(\mathrm{p}<0.05)^{6}$ and Dunne $C$ et al (2009) $(9.84 \pm 5.02$ hours in group 1 and $7.8 \pm 3.23$ hours in group 2$)(p<0.001)$ who demonstrated a significant short duration of labour in the induced group compared to spontaneous group in primipara subjects (Table 25). ${ }^{4}$

Table 25: Comparison of duration labour between the two groups (hour).

\begin{tabular}{|llll|}
\hline Author & \multicolumn{2}{l|}{ Primigravida } & P value \\
& $\begin{array}{l}\text { Group 1 } \\
\text { (mean } \pm \text { SD) }\end{array}$ & $\begin{array}{l}\text { Group 2 } \\
\text { (mean } \pm \text { SD) }\end{array}$ & \\
\hline $\begin{array}{l}\text { Smith LP } \\
\text { et al } \\
(1984)^{6}\end{array}$ & 10.9 & 8.6 & $<.05$ \\
\hline $\begin{array}{l}\text { Dunne C } \\
\text { et al } \\
(2009)^{4}\end{array}$ & $9.84 \pm 5.02$ & $7.8 \pm 3.23$ & $<.001$ \\
\hline $\begin{array}{l}\text { Present } \\
\text { Study }\end{array}$ & $9.1 \pm 4.42$ & $8 \pm 4.41$ & $<.001$ \\
\hline
\end{tabular}

The present study is comparable to Macer JA et al (1992) who demonstrated a significantly short mean duration of first stage of labour in the induced group compared to the spontaneous group among the primipara subjects $(7.2 \pm 3.2$ Vs 9.9 \pm 5.1 hours) $(\mathrm{p}=0.007)$ (Table 26). ${ }^{10}$

Table 26: Comparison of duration of first stage of labour between the two groups (hour).

\begin{tabular}{|llll|}
\hline Author & \multicolumn{2}{c|}{ Primigravida } & P value \\
& $\begin{array}{l}\text { Group 1 } \\
(\text { mean } \pm \text { SD) }\end{array}$ & $\begin{array}{l}\text { Group 2 } \\
(\text { mean } \pm \text { SD) }\end{array}$ & \\
\hline $\begin{array}{l}\text { Macer JA } \\
\text { et al } \\
(1992)^{10}\end{array}$ & $9.9 \pm 5.1$ & $7.2 \pm 3.2$ & .007 \\
\hline $\begin{array}{l}\text { Present } \\
\text { Study }\end{array}$ & $8.5 \pm 2.3$ & $7.4 \pm 1.6$ & $<.001$ \\
\hline
\end{tabular}

In the present study $35.4 \%$ of group 1 had caesarean delivery and $45.1 \%$ of group 2 had caesarean delivery. Primiparas demonstrated substantial effect of induction on caesarean delivery rate. Our findings were similar to observations made by Smith LP et al (1984) who reported $10.2 \%$ caesarean delivery rates in group 1 and $16.8 \%$ caesarean delivery rates in group 2 among the primipara subjects which was statistically significant. ${ }^{6}$ Dublin $\mathrm{S}$ et al (2000) reported $9.9 \%$ and $19.4 \%$ caesarean delivery rates in group 1 and group 2 among primiparas which was statistically significant. ${ }^{11}$ Dunne C et al (2009) also showed significant difference in caesarean delivery rates among primiparas $(6.6 \%$ and $13.3 \%$ in group 1 and group 2 respectively. ${ }^{4}$ Macer JA et al (1992) reported $22.1 \%$ caesarean delivery rate in group 1 and $33.8 \%$ in group 2 among primiparas but the findings were insignificant (Table 27). ${ }^{10}$

Table 27: Comparison of caesarean section rates between the two groups.

\begin{tabular}{|cccc|}
\hline Author & \multicolumn{2}{c|}{ Primigravida } & P value \\
\hline $\begin{array}{c}\text { Smith LP et } \\
\text { al (1984) }\end{array}$ & 10.2 & 16.8 & $<0.001$ \\
\hline $\begin{array}{c}\text { Macer JA et } \\
\text { al (1992) }\end{array}$ & 22.1 & 33.8 & 0.15 \\
\hline $\begin{array}{c}\text { Dublin S et al } \\
(2000)^{11}\end{array}$ & 9.9 & 19.4 & $1.77(\mathrm{RR})$ \\
\hline $\begin{array}{c}\text { Dunne C et al } \\
(2009)^{4}\end{array}$ & 6.6 & 13.3 & $<0.001$ \\
\hline Present study & 35.4 & 45.1 & 0.016 \\
\hline
\end{tabular}

The present study is contrary to study by Prysak $\mathrm{M}$ et al (1998) where fetal distress accounted for $0.9 \%$ and $1.5 \%$ of caesarean deliveries in group 1 and 2 and the difference was non-significant, dystocia was responsible for $7.6 \%$ and $3.5 \%$ of caesarean deliveries in the two groups $(\mathrm{p}<0.001){ }^{12}$ Seyb ST et al (1999) reported $1.7 \%$ and $4.2 \%$ incidence of caesarean delivery in group 1 and group 2 respectively for fetal distress which was nonsignificant and $6.1 \% \mathrm{Vs} 13.3 \%$ incidence of caesarean delivery due to dystocia in the two groups which was statistically significant $(\mathrm{p}<0.05) .{ }^{13}$ There were 18 caesareans $(8.8 \%)$ in our study which were done for failed induction. These were the cases that had Bishop score $\leq 5$ and did not have active labour even after using cervical ripening agents. Other studies have not observed such findings (Table 28).

The present study is comparable to Dunne C et al (2009) who also demonstrated no significant relationship in terms of post-partum hemorrhage between the two groups in the primipara and multipara subjects. ${ }^{4}$ Tylleskar $\mathbf{J}$ et al (1979) observed $339 \pm 242 \mathrm{ml}$ of blood loss in spontaneous group and $376 \pm 216 \mathrm{ml}$ of blood loss in induced group among primiparous subjects. The mean blood loss reported by them was more in the induced group but statistically non-significant. ${ }^{14}$ 
The present study is comparable to Macer JA et al (1992) who also reported no significant difference in the study and control groups as regards lacerations. ${ }^{10}$ Dunne $\mathrm{C}$ et al (2009) observed no difference between the two groups in cervical tears among primipara and multipara subjects and vaginal tears among primipara subjects. ${ }^{4}$ However they reported a statistically significant less perineal tears in the induced group in both primipara and multipara subjects ( $\mathrm{p}$ value 0.02 and 0.002 respectively) and vaginal tears among multipara subjects only $(\mathrm{p}=0.006)$. They had also observed a significantly longer second stage in both primipara and multipara electively induced groups. This extra time in the second stage could plausibly permit more accommodation by the bony pelvis and stretching of vaginal and pelvic tissues, factors that might have protected the perineum at delivery. Such findings were not observed in the present study.

The present study is comparable to Smith LP et al (1984) (7\% infants in group 1 and $6.8 \%$ in group 2 had 1 minute apgar score $<7)^{6}$, Macer JA et al (1992) (3.2\% in group 1 and $4.7 \%$ in group 2), ${ }^{10}$ Dunne C et al (2009) who reported the 1 minute apgar score separately among primipara (9.2\% infants in group 1 and $9.3 \%$ in group 2) and multipara $(6.3 \%$ in group 1 and $5 \%$ in group 2$) .{ }^{4}$ All these studies including the present study demonstrated no significant difference as regards the percentage of infants with apgar score $<7$ at 1 minute between the study and control groups.

The present study is comparable to Macer JA et al (1992) who reported incidence of 5 minute apgar $<7$ of $0.4 \%$ in group 1 and $0.8 \%$ in group $2,{ }^{10}$ Prysak $M$ et al (1998) $(0.2 \%$ in group 1 and $0.4 \%$ in group 2$),{ }^{12}$ Dublin $S$ et al (2000) $11(0.7 \%$ in group 1 and $0.9 \%$ in group 2 among primipara subjects, $0.4 \%$ in group 1 and $0.6 \%$ in group 2 among multiparous subjects). All these studies showed no significant relation in terms of percentage of infants with apgar score $<7$ at 5 minutes between the two study groups.

The present study is comparable to Macer JA et al (1992) (mean birth weight in group 1 was $3434 \pm 437 \mathrm{~g}$ compared to $3553+385 \mathrm{~g}$ in group 2), ${ }^{10}$ Seyb ST et al (1999) (mean weight in group 1 was $3400 \mathrm{~g}$ and in group 2 was 3548 $\mathrm{g}){ }^{13}$ Maslow AS et al (2000) (mean weight in group 1 was $3513 \pm 433 \mathrm{~g}$ and in group 2 was $3596 \pm 458 \mathrm{~g})$, ${ }^{15}$ Hoffman MK et al (2006) (mean weight in group 1 was $3373+434 \mathrm{~g}$ compared to $3438+401 \mathrm{~g}$ in group 2), ${ }^{16}$ Dunne $\mathrm{C}$ et al (2009) (mean weight in group 1 was $3413+412 \mathrm{~g}$ compared to $3522+403 \mathrm{~g}$ in group 2$).{ }^{4}$ These studies reported a significant difference as regards mean birth weight between group 1 and 2 being higher in induced group. According to Macer JA et al (1992) average birth weight in the induced group was higher and this did not appear to affect the delivery outcome (Table 29). ${ }^{10}$

However Cammu H et al (2002) (mean weight in group 1 was $3456 \pm 264 \mathrm{~g}$ and mean weight in group 2 was
$3461 \pm 264 \mathrm{~g}$ ) and Vrouenraets FPJM et al (2005) (mean weight in group 1 was reported to be $3360 \pm 470 \mathrm{~g}$ compared to $3435 \pm 542 \mathrm{~g}$ in group 2) have also reported slightly increased mean birth weight in group 2 but the difference was not statistically significant. ${ }^{9,17}$

The present study is comparable to study done by Prysak $M$ et al (1998) who reported an increased incidence of NICU admission in induced group compared to spontaneous group but statistically insignificant. ${ }^{12}$ Likewise, Cammu H et al (2002) also observed increased rate of NICU admission in induced group but insignificant. $^{17}$

Contrary to the present study Macer JA et al (1992) reported increased NICU admissions in the spontaneous group though statistically insignificant. ${ }^{10}$

In the present study, mean duration of stay in NICU in group 1 was 1.27 days and in group 2 was 1.14 days. The difference in hospital stay between the two groups was found to be non significant. The present study is comparable to study by Prysak M et al (1998) who reported mean of $2.2 \pm 1.8$ days of NICU admission in group 1 and $3.8 \pm 2.8$ days in group 2 and no statistical significance was reported. ${ }^{12}$ Thus it is concluded that induction of labour does not increase the mean duration of NICU length of stay

\section{CONCLUSIONS}

Primiparas who have spontaneous onset of labour the initial mean Bishop score is more compared to the subjects who have induced labour. The study demonstrated a significant increase in rate of caesarean section when Bishop score was $\leq 5(\mathrm{p}=0.047)$. Compared to those with spontaneous labour, primiparas with induced labour are more likely to have short duration of labour specially the first stage and higher incidence of caesarean delivery.

\section{Funding: No funding sources Conflict of interest: None declared Ethical approval: Not Required}

\section{REFERENCES}

1. Chua S, Arulkumaran S. Intrapartum care. Aust NZ J Obstet Gynecol. 1997;37:25-35.

2. Caughey AB, Sundaram V, Kaimal AJ, Cheng YW, GIenger A, Little SE et al. Maternal and Neonatal Outcomes of Elective Induction of Labor. Stanford: UCSF Evidences Based Practical centre (US). 2009 Report No. 09-E005.

3. Richardson A, Mmata C. Government Statistical Services for the Department of Health 2007. NHS Maternity Statistics, England. [Internet] 2005-2006.

4. Dunne C, Silva O, Schmidt G, Natale R. Outcomes of elective labour induction and elective caesarean section in low risk pregnancies between 37 and 41 
weeks gestation. J Obstet Gynecol Canada .2009, 31(12):1124-30.

5. Peregrine E, O`Brien P, Omar R, Jauniaux E. Clinical and ultrasound parameters to predict the risk of caesarean delivery after induction of labor. Obstet Gynecol. 2006;107:227-33.

6. Smith LP, Nagourney BA, McLean FH, Usher RH. Hazards and benefits of elective induction of labour. Am J Obstet Gynecol .1984;148:579.

7. Yudkin P, Frumar AM, Turnbull AC. A retrospective study of induction of labour. Br J Obstet Gynecol. 1979;86:257-65.

8. Johnson DP, Davis NR, Brown AJ. Risk of caesarean delivery after induction at term in nulliparous women with an unfavourable cervix. Am J Obstet Gynecol. 2003;188:1565-72.

9. Vrouenraets FPJM, Roumen FJME, Dehing CJG, Van den Akker ESA, Aarts MJB, Scheve EJT et al. Bishop score and risk of caesarean delivery after induction of labour in nulliparous women. Obstet Gynecol. 2005;105:690-7.

10. Macer JA, Macer CL, Chan LS. Elective induction versus spontaneous labour: A retrospective study of complications and outcome. Am J Obstet Gynecol. 1992;166:1690-7.

11. Dublin S, Rochelle ML, Kaplan RC, Watts DH, Critchlow CW. Maternal and Neonatal outcomes after induction of labor without an identified indication. Am J Obstet Gynecol .2000;183:986-94.
12. Prysak M, Castronova FC. Elective induction versus spontaneous labour: A Case-Control analysis of safety and efficacy. Obstet Gynecol .1998;92:47-52.

13. Seyb ST, Berka RJ, Socol ML, Dooley SL. Risk of caesarean delivery with elective induction of labour at term in nulliparous women. Obstet Gynecol. 1999;94:600-7.

14. Tylleskar J, Finnstrom O, Leijon I, Hedenskog S, Ryden G. Spontaneous labor and elective inductionA prospective randomised study. Acta Obstet Gynecol Scand. 1979;58:513-8.

15. Maslow AS, Sweeny AL. Elective induction of labour as a risk factor for caesarean delivery among low risk women at term. Obstet Gynecol. 2000;95: 917-22.

16. Hoffman M, Vahratian A, Sciscione A, Troendle J, Zhang J. Comparison of labour progression between induced and noninduced Multiparous women. Obstetrics and Gynaecology; 2006:107: 1029-1034. Cammu H, Martens G, Ruyssinck G, Amy JJ. Outcome after elective labour induction in nulliparous women: A matched cohort study. Am J Obstet Gynecol. 2002;186:240-4.

Cite this article as: Sood P, Sood A, Sood R, Sood $\mathrm{S}$, Sood M. To ascertain any differences in fetomaternal outcomes in induced and spontaneous labour among primiparous women delivering at term without an identified indication for induction. Int $\mathbf{J}$ Reprod Contracept Obstet Gynecol 2016;5:121-8. 